\title{
Ordered mesoporous tin oxide and tin phosphate synthesized by nanocasting strategy
}

\author{
Paulina Półrolniczak • Stanisław Kowalak
}

Published online: 4 November 2010

(C) The Author(s) 2010. This article is published with open access at Springerlink.com

\begin{abstract}
Ordered mesoporous tin oxide and tin phosphate were successfully synthesized via two-step nanocasting route. The SBA-15 silica and CMK-3 carbon were used as hard templates. Powder X-ray diffraction, nitrogen adsorption and transmission electron microscopy confirmed hexagonal mesoporous structure of resulted products. Mesoporous tin oxide indicated crystalline walls (cassiterite). The mesoporous products showed considerable catalytic activity in propan-2-ol decomposition. The tin oxide led the dehydrogenation towards acetone, while mesoporous tin phosphate exhibited activity of acid sites resulting in dehydration to propene.
\end{abstract}

Keywords Mesoporous tin oxide - Mesoporous tin phosphate - Nanocasting - Ordered mesoporous structure

\section{Introduction}

Since the discovery of ordered mesoporous materials M41S in 1992 [1], silica mesoporous molecular sieves, possessing remarkably large internal surface areas and narrow pore size distributions have become a widely explored due to the potential application of these materials to use as adsorbents, catalysts and catalyst supports. In recent years, the supramolecular templating pathway has been applied for the synthesis of mesostructured metal oxides such as $\mathrm{SnO}_{2}$ [2], $\mathrm{TiO}_{2}, \mathrm{ZrO}_{2}$. More recently, several mesostructured and mesoporous aluminophosphates with lamellar or hexagonal structures have been investigated using cationic, anionic

P. Półrolniczak $(\bowtie) \cdot S$. Kowalak

Faculty of Chemistry, A. Mickiewicz University,

Grunwaldzka 6, 60-780 Poznań, Poland

e-mail: paulap@amu.edu.pl and non-ionic templates [3]. Despite the great progress in syntheses of novel ordered mesoporous materials, only a few preparative approaches have been reported on the synthesis of mesoporous transition metal phosphates, including iron phosphate, tin phosphate [4, 5], titanium phosphate [6] and zirconium phosphate. Most of these mesoporous phosphates are prepared in hydrothermal conditions with the aid of cationic surfactants. The main drawback of non-silica mesoporous materials is that these structures do not withstand a removal of the templates by calcination or even milder procedures [7]. A hydrolysis and polymerization of the transition metal precursors are not easy to control and the resulting products often show limited ordering and are thermally unstable [8]. The use of mesoporous solid materials as hard template, which cast the structure faithfully to the final product [9, 10] seems very promising alternative.

Our earlier attempts to prepare mesoporous tin oxide and tin-phosphate systems with conventional templates resulted mostly in unstable products, whose pore ordering deteriorated markedly upon thermal removal of the template [11]. The ordered porous tin oxide or tin-phosphate materials could appear very useful as potential catalysts or electrode materials in Li-ion batteries [12]. Here, we present a procedure based on the two-step nanocasting route to prepare stable ordered mesoporous tin oxide and tin phosphate, which are first ordered tin materials obtained this way.

\section{Experimental}

Ordered mesoporous tin oxide and tin phosphate were synthesized via two-step replication method using first the mesoporous silica SBA-15 for preparing the mesoporous 
carbon CMK-3 and the latter as matrix for the formation of tin bearing mesoporous products. Mesoporous SBA-15 silica was synthesized according to literature procedure [13], with triblock copolymer Pluronic P123 as structure directing agent and tetraethylorthosilicate (TEOS) as silica source. The calcined SBA-15 material was then used as template for the preparation of mesoporous carbon. Mesoporous carbon CMK-3 was prepared by means of procedure reported by S. Jun et al. [14]. Calcined SBA-15 material was impregnated twice with aqueous solution of sucrose containing sulfuric acid. The mixture was then heated for $6 \mathrm{~h}$ at $100{ }^{\circ} \mathrm{C}$ and then at $160{ }^{\circ} \mathrm{C}$ for additional $6 \mathrm{~h}$. The carbonization was completed by pyrolysis at $800{ }^{\circ} \mathrm{C}$ for $1 \mathrm{~h}$. The silica template was dissolved with $5 \%$ HF solution. The resulted mesoporous carbon CMK-3 was afterwards employed as a porogeneous agent for assembling the mesoporous tin-phosphate materials. In a typical syntheses $0.5 \mathrm{~g}$ of $\mathrm{CMK}-3$ was dispersed in alkaline aqueous solution ( $\mathrm{pH} \sim 8$ ), containing $0.5 \mathrm{~g}$ of $\mathrm{SnCl}_{4}$. $5 \mathrm{H}_{2} \mathrm{O}$ in the case of ordered $\mathrm{SnO}_{2}$ synthesis and $0.5 \mathrm{~g}$ of $\mathrm{SnCl}_{4} \cdot 5 \mathrm{H}_{2} \mathrm{O}$ with $0.14 \mathrm{~g}$ of $85 \% \mathrm{H}_{3} \mathrm{PO}_{4}$ in $\mathrm{SnPO}$ synthesis. The P/Sn molar ratio was 1 (estimated by ICP OES analysis). The mixture was stirred for $3 \mathrm{~h}$ at ambient temperature and heated for $3 \mathrm{~h}$ at $40{ }^{\circ} \mathrm{C}$ followed by $96 \mathrm{~h}$ at $80{ }^{\circ} \mathrm{C}$. Finally, carbon matrix was burned out at $500{ }^{\circ} \mathrm{C}$ in air for $4 \mathrm{~h}$. The carbon content after calcination was $0.2 \%$, determined by elemental analysis.

The nature of catalytic active sites was estimated in model test reaction for propan-2-ol decomposition. Propan2-ol decomposition was conducted at $230{ }^{\circ} \mathrm{C}$ in a pulse microreactor combined with GC (Chrom 5 equipped with TCD as a detector). The powder calcined samples $(0.015 \mathrm{~g})$ were activated in helium stream at $400{ }^{\circ} \mathrm{C}$ for $30 \mathrm{~min}$. prior the catalytic test. The volume of injected substrates was $1 \mu \mathrm{L}$.

\section{Results and discussion}

Figure 1 shows the powder low-angle X-ray diffraction patterns of a mesoporous tin phosphate as well as those of the parent SBA-15 and CMK-3 which consecutively served as templates. The smaller intensity of the SnPO 100 reflection indicates some decrease in structure ordering in comparison to hexagonal SBA-15 structure. No reflection is observed in the wide-angle region (not shown). In the case of ordered mesoporous tin oxide, the low-angle XRD exhibits intense reflections, moreover the wide-angle XRD confirms the crystalline cassiterite $\left(\mathrm{SnO}_{2}\right)$ structure (Fig. 2).

The transmission electron microscopy (TEM) images show an intact long-range order with hexagonal symmetry in the resultant tin oxide (Fig. 3) and in tin phosphate (Fig. 4).

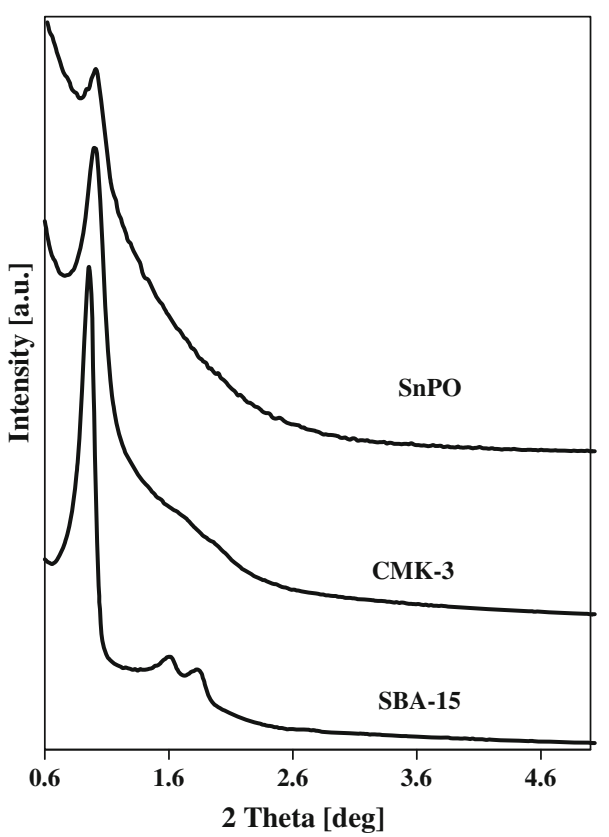

Fig. 1 XRD patterns of $S n P O$ and $S B A-15, C M K-3$ matrices

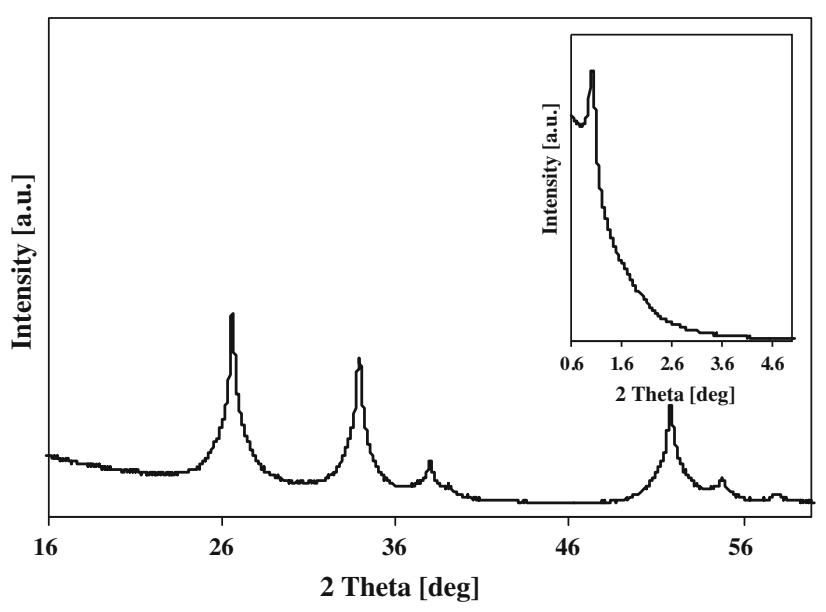

Fig. 2 Wide-angle and low-angle (inset) XRD patterns of ordered mesoporous $\mathrm{SnO}_{2}$

The mesoporous nature of the products is confirmed by nitrogen adsorption. The isotherms show the shape reminding the IV type of isotherm, characteristic for mesoporous materials (Fig. 5). The BJH pore diameters, the specific BET surface areas and pore volumes of all materials, SBA-15, CMK-3, $\mathrm{SnO}_{2}$ and tin phosphate are given in Table 1, (Fig. 6).

The substantial differences in the specific surface area and total pore volumes could be consistent with the fact that the densities of the respective materials increase in order $\mathrm{C}<\mathrm{SiO}_{2}<\mathrm{SnO}_{2}$. However, the above-mentioned partial loss of structural order also accounts for the lower surface areas of mesoporous tin oxide and tin phosphate as compared to that of SBA-15. 
Fig. 3 TEM images of mesoporous tin oxide
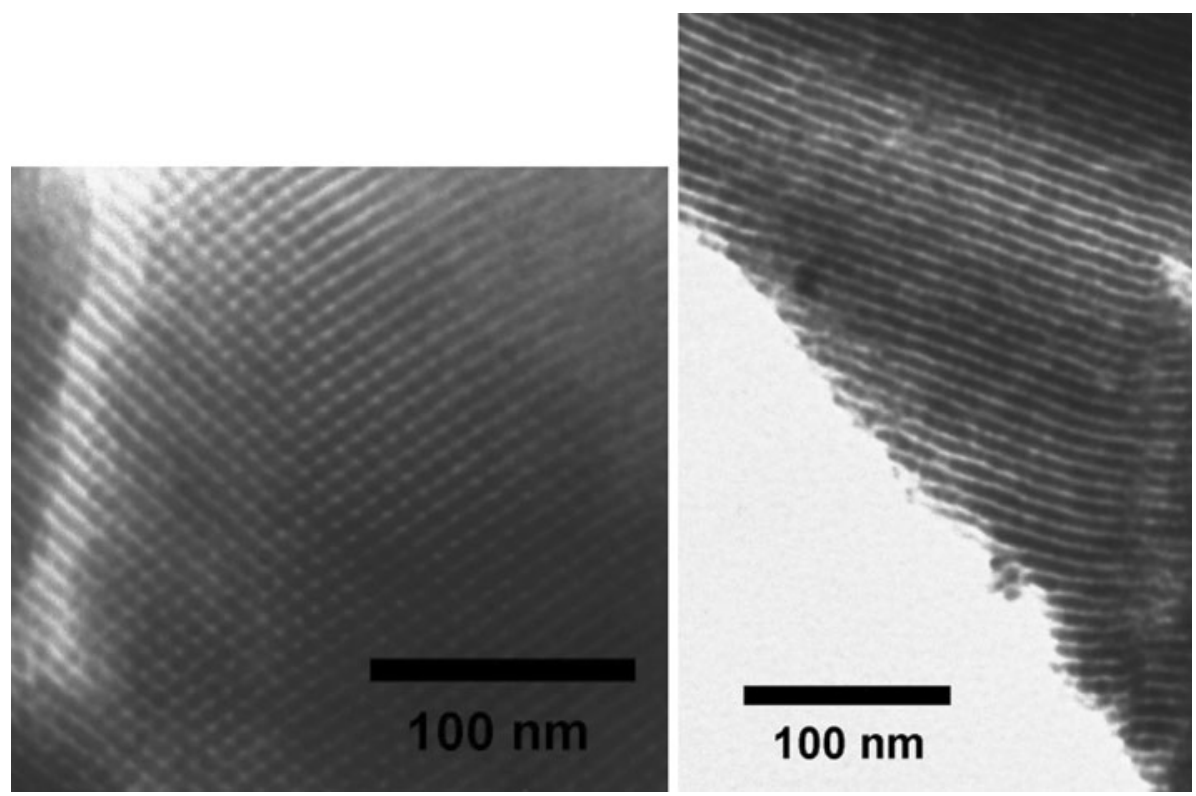

Fig. 4 TEM images of mesoporous tin phosphate
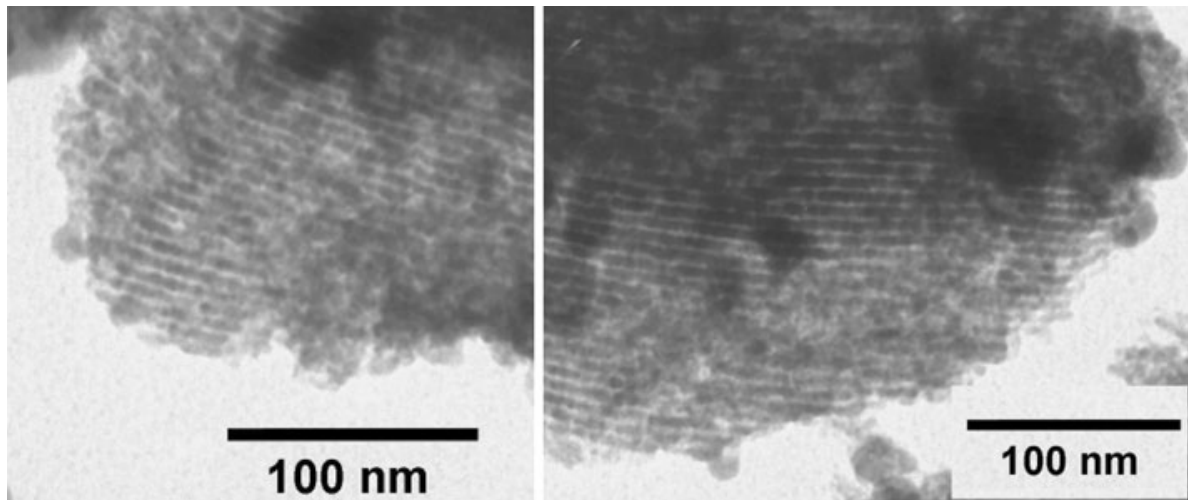

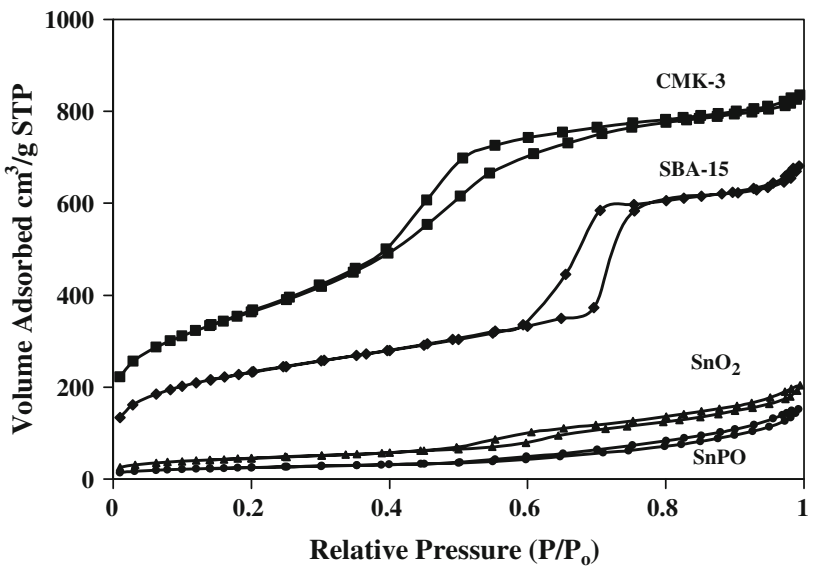

Fig. 5 Nitrogen physisorption isotherms of $S B A-15, C M K-3$ and synthesized tin materials

The synthesized materials exhibit some catalytic activity in propan-2-ol decomposition. The prepared tin oxide shows considerable catalytic activity with high ( $>90 \%)$
Table 1 Physisorption data of SBA-15, CMK-3 and tin materials

\begin{tabular}{lcll}
\hline Material & $\begin{array}{l}\text { Surface area } \\
\text { BET }\left[\mathrm{m}^{2} / \mathrm{g}\right]\end{array}$ & $\begin{array}{l}\text { Pore volume } \\
{\left[\mathrm{cm}^{3} / \mathrm{g}\right]}\end{array}$ & $\begin{array}{l}\text { Pore diameter } \\
{[\mathrm{nm}]}\end{array}$ \\
\hline $\mathrm{SBA}-15$ & 740 & 0.86 & 7.1 \\
$\mathrm{CMK}-3$ & 1,306 & 1.26 & 3.8 \\
$\mathrm{SnO}_{2}$ & 165 & 0.3 & 6.5 \\
$\mathrm{SnPO}$ & 91 & 0.2 & 8.6 \\
\hline
\end{tabular}

selectivity towards acetone confirming the presence of redox active sites typical of $\mathrm{SnO}_{2}$. The mesoporous tin phosphate leads the reaction towards propene which results from action of acid sites. An ordering of mesoporous system in the investigated samples does not improve the catalytic efficiency. As a matter of facts the conversions recorded under the same conditions over the samples prepared with conventional templates who lost the pore ordering upon the template removal was always markedly 


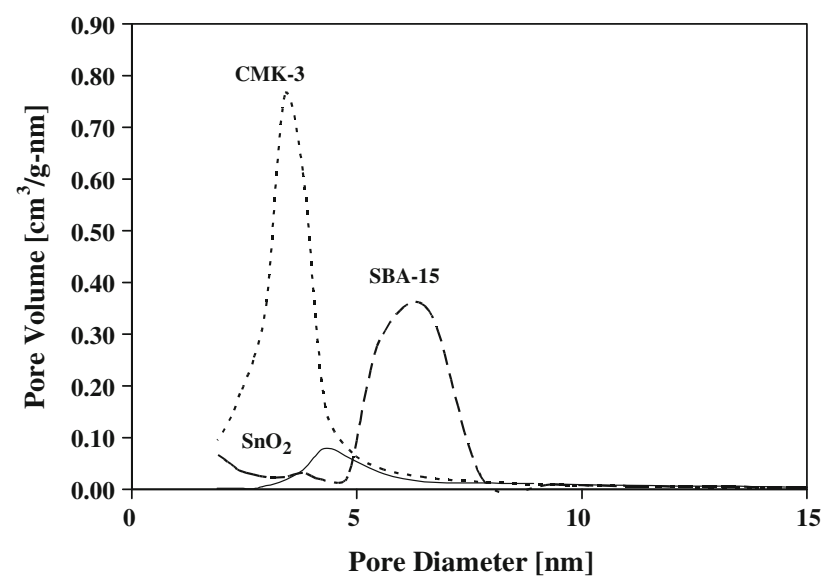

Fig. 6 Pore size distributions of $S B A-15, C M K-3$ and synthesized tin materials

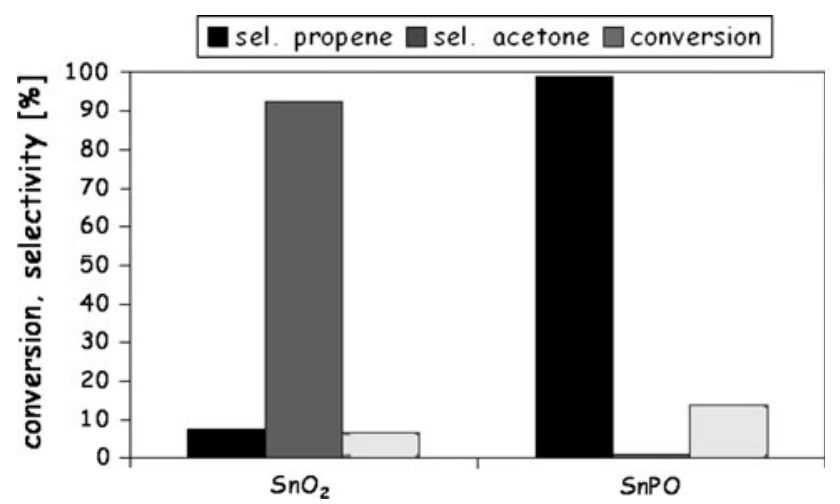

Fig. 7 Propan-2-ol decomposition test reaction

higher $(\sim 70 \%)$. Perhaps, the advantage of pore ordering could be more conspicuous in catalytic reactions of bulkier molecules, where reagent diffusion is an important factor (Fig. 7).

\section{Conclusions}

The presented results indicated that the relatively well ordered mesoporous tin oxide and tin phosphate can be prepared by nanocasting with the aid of hard templates (CMK-3 carbon and SBA-15 silica). Contrary to the products obtained by hydrothermal synthesis with conventional templates the resulting products show a high thermal stability and they withstand severe treatment at $500{ }^{\circ} \mathrm{C}$ (in order to burn out the CMK-3 carbon template). Although the adopted nanocasting method is more complex than the conventional hydrothermal methods, it showed for the first time a possibility to obtain temperature resistant ordered mesoporous tin oxide and tin phosphate. It is worth underlining that resultant tin oxide is a hybrid material consisting of crystalline walls.

Acknowledgments We thank the Polish Ministry of Science and Higher Education for financial support under the project N N204 119238.

Open Access This article is distributed under the terms of the Creative Commons Attribution Noncommercial License which permits any noncommercial use, distribution, and reproduction in any medium, provided the original author(s) and source are credited.

\section{References}

1. C.T. Kresge, M.E. Leonowicz, W.J. Roth, J.C. Vartuli, J.S. Beck, Nature 359, 710 (1992)

2. Y.-D. Wang, C.-L. Ma, X.-D. Sun, H.-D. Li, Mater. Lett. 51, 285 (2001)

3. M. Tiemann, M. Fröba, G. Rapp, S.S. Funari, Chem. Mater. 12, $1342(2000)$

4. N.K. Mal, S. Ichikawa, M. Fujiwara, Chem. Commun. 2002, 112

5. C. Serre, A. Auroux, A. Gervasini, M. Hervieu, G. Ferey, Angew. Chem. Int. Ed. 41, 1594 (2002)

6. A. Bhaumik, S. Inagaki, J. Am. Chem. Soc. 123, 691 (2001)

7. A. Sayari, P. Liu, Microporous Mater. 12, 149 (1997)

8. H. Yang, D. Zhao, J. Mater. Chem. 15, 1217 (2005)

9. J. Roggenbuck, M. Tiemann, J. Am. Chem. Soc. 127, 1096 (2005)

10. M. Kang, S.H. Yi, H.I. Lee, J.E. Yie, J.M. Kim, Chem. Comm. 8, 1944 (2002)

11. P. Półrolniczak, S. Kowalak, Stud. Surf. Sci. Catal. 174 A, 405 (2008)

12. J.-G. Lee, D. Son, Ch. Kim, B. Park, J. Power Sources 172, 908 (2007)

13. D. Zhao, Q. Huo, J. Feng, B.F. Chmelka, G.D. Stucky, J. Am. Chem. Soc. 120, 6024 (1998)

14. S. Jun, S.H. Joo, R. Ryoo, M. Kruk, M. Jaroniec, Z. Liu, T. Ohsuna, O. Terasaki, J. Am. Chem. Soc. 122, 10712 (2000) 